\title{
Prevalencia de diagnósticos de enfermeria en el programa de detección temprana de cáncer de cérvix
}

\author{
Prevalence of nursing diagnoses in the early \\ cervical cancer detection program
}

\author{
Iliana-Milena Ulloa ${ }^{1}$; Luz Dalia Valbuena-Duran²
}

Forma de citar: Ulloa IM, Valbuena LD. Prevalencia de diagnósticos de enfermería en el programa de detección temprana de cáncer de cérvix. Salud UIS. 2020; 52(4): 382-390. doi: https://doi.org/10.18273/revsal.v52n4-2020005 (c) (i)

\begin{abstract}
Resumen
Introducción: el cáncer de cérvix representa un problema de salud pública a nivel mundial, al constituirse en la tercera causa de mortalidad por neoplasias malignas en la mujer Objetivo: determinar la prevalencia de los diagnósticos de enfermería en usuarias del programa de detección temprana de cáncer de cérvix, en una institución de salud de primer nivel de atención del municipio de Girón, Santander. Materiales y métodos: estudio de corte transversal. La muestra estuvo conformada por 85 usuarias atendidas por estudiantes tercer nivel de enfermería durante la práctica de Cuidado de la Salud de la Mujer. Se realizó valoración focalizada del estado de salud a partir del Formato de Valoración de la Escuela de Enfermería de la Universidad Industrial de Santander; e información registrada en el Sistema de Información de Prácticas Clínicas y Comunitarias Estudiantiles (SIPCE). Resultados: los diagnósticos de mayor prevalencia fueron Disposición para mejorar los conocimientos con un 35,29\%, Conocimientos deficientes con el 32,29\% y Disposición para mejorar el autocuidado con 20,00\%. Entre los de menor prevalencia se encuentran mantenimiento ineficaz de la salud en un 2,35\%, Temor y Riesgo de infección con 4,71\% cada uno. Conclusión: la identificación de los diagnósticos más frecuentes en usuarias del programa de detección temprana de cáncer de cérvix, es una propuesta para que estudiantes y profesionales de enfermería lleven a cabo planes de cuidado, mediante el uso del lenguaje estandarizado en el ejercicio de la práctica de enfermería.
\end{abstract}

Palabras clave: Citología; Diagnóstico de enfermería; Neoplasias del cuello uterino; Prevalencia; Programas nacionales de salud.

\footnotetext{
Abstract

Introduction: Cervical cancer represents a public health problem worldwide, as it is the second leading cause of mortality due to malignant neoplasms in women. Objetive: To determine the prevalence of nursing diagnoses in users of the cervical cancer early detection program, in a first level health care institution in the municipality of Girón,

1. Universidad Industrial de Santander. Bucaramanga, Colombia.

Correspondencia. Iliana Milena Ulloa Sabogal. Dirección: Facultad de Salud, Cra. 32 29-31 Ed Eloy Valenzuela, Piso 5 Bucaramanga. Teléfono:+57 76344000 Ext 3005. Correo electrónico: imulloa@uis.edu.co. 
Santander. Materials and methods: Cross section study. The sample consisted of 85 users attended by third level nursing students during the practice of Women's Health Care. A focused assessment of the state of health was carried out using the Valuation Form of the Nursing School of the Universidad Industrial de Santander, and information registered in the Information System of Clinical and Community Student Practices (SIPCE). Results: The most prevalent diagnoses were Disposition to improve knowledge with 35.29\%, deficient knowledge with 32.29\% and willingness to improve self-care with $20.00 \%$. Among the lowest prevalence are Ineffective maintenance of health at $2.35 \%$, Fear and Risk of infection with $4.71 \%$ each. Conclusion: The identification of the most frequent diagnoses in users of the cervical cancer early detection program is a proposal for students and nursing professionals to carry out care plans, through the use of standardized language in the exercise of nursing practice.

Keywords: Citology; Nursing diagnosis; Uterine cervical neoplasms; Prevalence; National health programs.

\section{Introducción}

El cáncer de cérvix es un importante problema de salud pública en todo el mundo ${ }^{1}$, con una estimación anual de 500000 casos nuevos, de los cuales aproximadamente el $83 \%$ se presentan en países en desarrollo $^{2}$. De acuerdo con la Organización Mundial de la Salud, la tasa estandarizada a nivel mundial es de 15,2 x 100000 mujeres. Las mayores incidencias se presentan en las regiones de África, sureste de Asia y las Américas, con tasas de 30,7, 24,4 y 15,3 x 100000 mujeres, respectivamente. En el mundo, las muertes por cáncer de cérvix ocupan el tercer lugar, con un total de 31712 defunciones, lo cual representa el 8,22\% de las muertes ocurridas por neoplasias malignas, con una tasa de mortalidad estandarizada de 7,8 x 100000 mujeres $^{3}$.

En Colombia, el cáncer de cérvix permanece como la principal causa de mortalidad y la segunda causa de incidencia de cáncer entre las mujeres, a pesar de la existencia en el país de programas de detección temprana en las últimas tres décadas ${ }^{4}$. En el año 2018 se presentó una incidencia de 18,4 casos nuevos por cada 100000 mujeres $^{5}$. En el mismo año la tasa de mortalidad fue de 10 defunciones por 100000 mujeres $^{4}$.

Considerando esta realidad el Ministerio de Salud y Protección Social (MSPS) en Colombia, ha establecido normativas para las actividades orientadas a la detección precoz, diagnóstico y tratamiento oportuno de lesiones precursoras del cáncer de cérvix; de esta manera, la Resolución 412 de 2 000, implementa la Norma Técnica para la detección temprana y Guía de atención de lesiones preneoplásicas de cuello uterino ${ }^{6}$. Posteriormente, la Guía de Práctica Clínica para la detección y manejo de lesiones precancerosas de cuello uterino-2014 ${ }^{7}$. Así mismo, en el marco de la Resolución 429 de 2016, se adoptó la Política de Atención Integral en Salud (PAIS), en la que se establece el Modelo Integral de Atención en Salud (MIAS), donde uno de sus componentes es la regulación de Rutas Integrales de Atención en Salud (RIAS). Una de las RIAS definidas, es la de cáncer de cuello uterino, en la cual se recogen las recomendaciones dadas por la Guía de Práctica Clínica ${ }^{8}$. Adicionalmente, el Plan Decenal de Salud Pública Colombiano (PDSP 2012-2021), ha establecido dentro de sus metas reducir la tasa de mortalidad a 5,5 muertes por 100.000 mujeres en 2021; alcanzar coberturas de tamización con citología de cáncer de cérvix en el $80 \%$ de la población objeto (mujeres de 21 - 69 años); e incrementar la proporción de casos de cáncer de cérvix in situ detectados oportunamente, en un $80 \%{ }^{9}$.

En este contexto, el cáncer de cérvix representa un desafío para el sistema y los profesionales de la salud, especialmente en aquellos campos en los que la intervención está marcada por la Atención Primaria en Salud (APS), incluyendo las acciones de promoción, prevención y atención de bajo riesgo ${ }^{10}$.

A nivel mundial, el profesional de enfermería es un líder en el marco de la estrategia de atención primaria en salud, su rol se fundamenta en el desarrollo de acciones que van desde la planeación, gestión, promoción de la salud, prevención de la enfermedad, educación sobre derechos y deberes, motivación a la participación de la comunidad, y otras, que contribuyen a mejorar salud y bienestar de la población ${ }^{11}$. Por consiguiente, la esencia del quehacer de enfermería es el cuidado integral del ser humano, y para lograrlo cuenta con el proceso de atención de enfermería, en el cual el diagnóstico es el eje central ${ }^{12}$.

Un diagnóstico de enfermería es una respuesta que describe la necesidad humana (estado de salud real o potencialmente alterado) de una persona, familia, grupo o comunidad, que le permite al enfermero identificar y seleccionar las intervenciones destinadas a conseguir los resultados sobre los cuales enfermería tiene responsabilidad; dichas intervenciones buscan mantener el estado de salud, reducir o prevenir alteraciones ${ }^{13}$. 
En este sentido, la identificación de diagnósticos de enfermería proporcionará las bases no sólo para fortalecer las estrategias e intervenciones encaminadas a la prevención, detección temprana, seguimiento y control del cáncer de cérvix, sino la utilización de un lenguaje estandarizado con el que enfermería pueda comunicar su propio cuidado.

De esta manera, el objetivo del presente estudio fue determinar la prevalencia de los diagnósticos de enfermería en usuarias del programa de detección temprana de cáncer de cérvix, en una entidad de salud de primer nivel de atención del municipio de Girón, Santander.

\section{Materiales y métodos}

Estudio descriptivo de corte transversal, en mujeres asistentes al Programa de detección temprana de cáncer de cérvix, en una institución de salud de primer nivel de atención en el municipio de Girón, Santander, entre junio y agosto del año 2016. La población estuvo conformada por 369 mujeres asistentes en el Programa de detección de cáncer de cérvix, en el período de junio y agosto, de las cuales 85 fueron seleccionadas por conveniencia, durante la práctica formativa cuidado de la salud de la mujer del programa de enfermería de la Universidad Industrial de Santander.

Los criterios de inclusión establecidos fueron: usuaria asistente al programa de detección temprana de cáncer de cérvix de la institución de salud, atendida por un estudiante de III nivel de programa de enfermería, que aceptara la participación y firma del consentimiento informado escrito de la institución de salud; el criterio de exclusión fue usuaria con incapacidad cognitiva o de comunicación verbal para responder el formato de valoración.

La recolección de la información fue llevada a cabo por 20 estudiantes y una profesora del programa de enfermería; se realizó a cada usuaria una valoración que incluyó la entrevista y el examen físico, utilizando como instrumento el "Formato para valoración del estado de salud" basado en la Taxonomía II de NANDA I (North American Nursing Diagnosis Association)," el cual fue diseñado, evaluado y validado por un grupo de investigadores de la Escuela de Enfermería de la Universidad Industrial de Santander ${ }^{12,14,15}$. El proceso de valoración contempló 14 variables focalizadas en los dominios de promoción de la salud, actividad y reposo, percepción-cognición, seguridad-protección $\mathrm{y}$ afrontamiento y tolerancia al estrés; preguntas que facilitaron la identificación de las características definitorias y factores de riesgo para la selección de los diagnósticos de enfermería. Adicionalmente, el formato incluyó la valoración del dominio de sexualidad que permitió establecer 10 variables clínicas relacionadas con salud sexual y reproductiva; además se incluyeron las variables sociodemográficas para la caracterización de la población.

La entrevista fue realizada por un estudiante de enfermería durante el tiempo de espera de la consulta, tiempo aproximado de 25-30 minutos, posteriormente, durante la consulta con la docente fueron confirmados los datos de la entrevista y llevado a cabo el examen físico.

Para la identificación de los diagnósticos, se utilizaron los Diagnósticos Enfermeros de la NANDAI 2015-2017, en la que se encuentran 235 diagnósticos que describen los problemas de salud reales o potenciales, y a partir de sus características definitorias o factores relacionados permiten confirmar la presencia del diagnóstico en la persona, familia o comunidad que se encuentran bajo el cuidado de enfermería ${ }^{16}$. Los diagnósticos elegidos por el estudiante fueron analizados y priorizados por la docente y el estudiante, con el propósito de seleccionar el diagnóstico principal.

Los datos obtenidos en la valoración y el diagnóstico principal con sus características definitorias o factores de riesgo, fueron registrados en el Formato del Sistema de Información de Prácticas Clínicas y Comunitarias Estudiantiles (SIPCE) de la Escuela de Enfermería de la Universidad Industrial de Santander.

Para el análisis de la información los datos obtenidos fueron ingresados en una base de datos diseñada en el programa Microsoft Office Excel 2010, posteriormente se exportaron los datos para su análisis estadístico en el programa de STATA $14^{17}$. Se realizó análisis descriptivo de las características sociodemográficas de las participantes de estudio; se evaluaron distribuciones de frecuencia y medidas de tendencia central; la prevalencia de los diagnósticos de enfermería de interés fue calculada con sus respectivos intervalos de confianza del $95 \%$.

\section{Resultados}

\section{Características sociodemográficas}

En la caracterización de las 85 participantes, se puede resaltar que la mediana de edad fue de 39,5 años, con una edad mínima de 15 años y una máxima de 71 años; el $35,29 \%$ de ella eran solteras y el $39,59 \%$ vivían en 
unión libre; el 74,12\% las participantes se ubicaban en el estrato socioeconómico I; la mediana de años de escolaridad fue de 6,5 años cursados y aprobados, con un mínimo de 1 y máximo de 13 años; en el 58,82\% su ocupación era el hogar/ama de casa y el $100 \%$ se encontraban afiliadas al régimen subsidiado en salud.

Tabla 1. Caracterización del perfil sociodemográfico de las usuarias del Programa de Detección Temprana de Cáncer de Cérvix. Girón- Santander, 2016

\begin{tabular}{lc}
\hline Características sociodemográficas & n (\%) \\
\hline Estado Civil & \\
Soltera & $30(35,29)$ \\
Casada & $19(22,35)$ \\
Unión libre & $26(30,59)$ \\
Divorciada / Separada & $4(4,71)$ \\
Viuda & $6(7,06)$ \\
& \\
Estrato Socioeconómico & \\
0 & \\
1 & $1(1,18)$ \\
2 & $63(74,12)$ \\
3 & $17(20,00)$ \\
& $4(4,71)$ \\
Nivel de escolaridad & \\
Primaria Incompleta & \\
Primaria Completa & $19(22,35)$ \\
Básica secundaria & $24(28,23)$ \\
Secundaria & $19(22,35)$ \\
& $23(27,05)$ \\
Ocupación & \\
Estudiante & \\
Empleada & $5(5,88)$ \\
Hogar/ Ama de casa & $16(18,82)$ \\
\hline & $14(16,47)$ \\
& $50(58,82)$ \\
\hline & \\
& \\
&
\end{tabular}

\section{Características en salud sexual y reproductiva}

En relación con los antecedentes en salud sexual y reproductiva, se encontró un $16,47 \%$ de las usuarias eran nuligravidas y el $83,53 \%$ restante había tenido uno o más embarazos; el 41,18 \% eran nulíparas y el $58,82 \%$ tenía una multiparidad entre 1 y 12 partos; el $24,71 \%$ al menos una cesárea y el $11,76 \%$ presentó al menos un aborto. El método anticonceptivo más usado fue el definitivo femenino con $24,71 \%$, la utilización del preservativo en tan solo un $14,12 \%$ y un $21,18 \%$ no utilizaba ningún tipo de anticonceptivo.
Tabla 2. Características en salud sexual y reproductiva de las usuarias del Programa de Detección Temprana de Cáncer de Cérvix. Girón- Santander, 2016

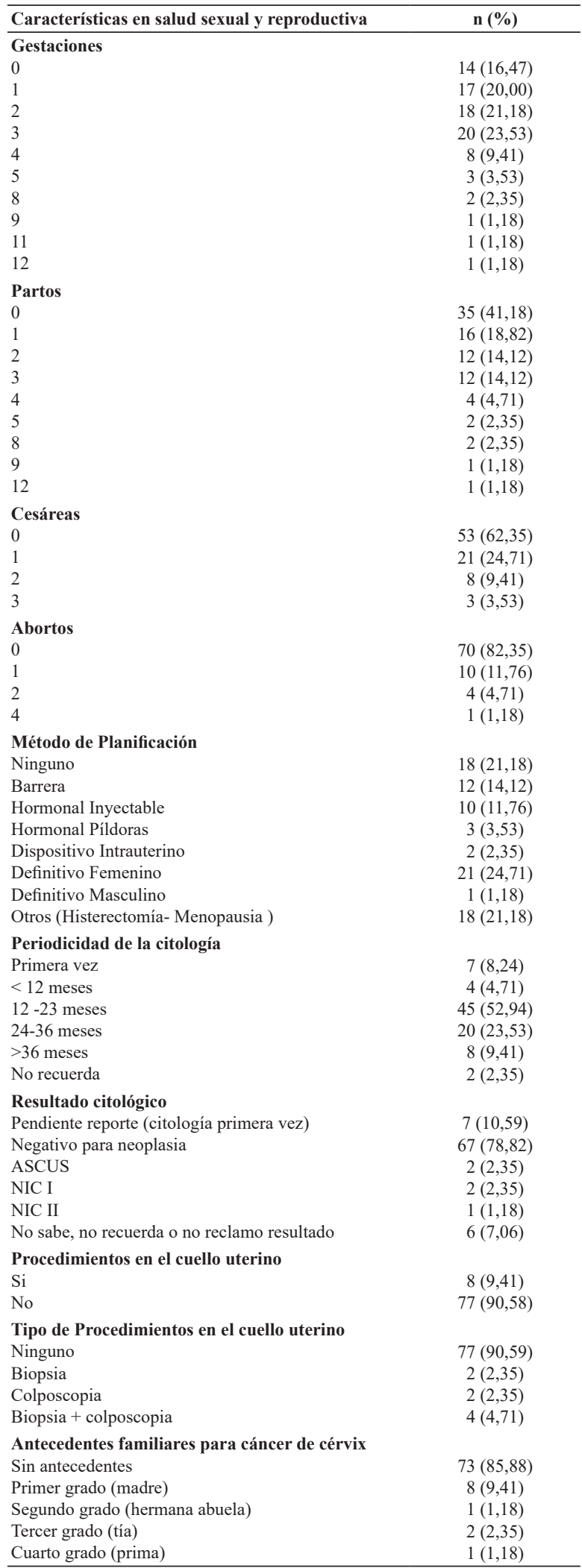


Con respecto a la citología cervicouterina el $44,71 \%$ de las mujeres reconocen la citología, como un medio para identificar tempranamente el cáncer de cérvix, sin embargo el 55,29\% restante desconoce el objetivo principal de la citología, y la relaciona como un medio diagnóstico para otras patologías, entre ellas vaginosis, infecciones de transmisión sexual, miomas uterinos o quistes en el ovario; el 92,94\% de las mujeres participantes habían realizado por lo menos una citología cervicouterina y el 7,06\% manifestó ser la primera vez que asistía al programa; de acuerdo a la periodicidad de la toma un $4,71 \%$ realizó la citología con un intervalo de tiempo inferior a un año, el $52,94 \%$ de las mujeres se realizan la citología entre 1 año y 23 meses, un 23,53\% entre 2 y 3 años y un $11,76 \%$ mayor a 3 años o no recuerda; el $78,82 \%$ de las mujeres refirieron reportes normales en sus citologías anteriores (negativo para neoplasia) y el 5,88\% manifestaron alteraciones en alguno de sus resultados citológicos, entre ellos, ASCUS (células escamosas de significado indeterminado) NIC I (Neoplasia Intraepitelial de Bajo Grado) y NIC II (Neoplasia Intraepitelial de Alto Grado) y el 8,23\% no sabe, no recuerda o no reclamo su resultado citológico; el $9,41 \%$ de las mujeres había requerido algún procedimiento por lesiones tipo inflamación, úlceras o pólipos cervicales, o como medio diagnóstico por alteraciones en la última o de citologías anteriores; el
14,12\% había tenido al menos un antecedente familiar para cáncer de cérvix en primero, segundo, tercer o cuarto grado de consanguineidad.

\section{Prevalencia de los diagnósticos de enfermería}

En relación con el objetivo del estudio los diagnósticos de enfermería con mayor prevalencia entre las usuarias fueron Disposición para mejorar los conocimientos con un $35,29 \%$, seguido de conocimientos deficientes en un $32,29 \%$, con las características definitorias de conocimiento insuficiente y expresa deseo de mejorar en el aprendizaje respectivamente, y el diagnóstico Disposición para mejorar el autocuidado en un $20,00 \%$ con las características definitorias de deseos de aumentar el autocuidado y expresa deseos de aumentar el conocimiento de las estrategias para el autocuidado; de otra parte los diagnósticos con menor prevalencia fueron Mantenimiento ineficaz de la salud con un $2,35 \%$ con las características de conocimiento insuficiente sobre prácticas básicas de salud, desinterés para mejorar las conductas de salud y patrón de falta de conducta de búsqueda de salud, Temor con la característica identifica el objeto de temor y riesgo de infección con el factor de Riesgo conocimiento insuficiente para evitar la exposición a agentes patógenos cada uno con un 4,71\%. (Tabla 1)

Tabla 3. Prevalencia de diagnósticos de enfermería en usuarias de las usuarias del Programa de Detección Temprana de Cáncer de Cérvix. Girón- Santander, 2016

\begin{tabular}{lccc}
\hline \multicolumn{1}{c}{ Etiqueta Diagnóstica } & $\mathbf{n ~ ( \% )}$ & Desviación Estándar & IC- 95\% \\
\hline Conocimientos deficientes & $28(32,94)$ & 0,050 & $0,231-0,439$ \\
Disposición para mejorar los conocimientos & $30(35,29)$ & 0,051 & $0,252-0,464$ \\
Disposición para mejorar el autocuidado & $17(20,00)$ & 0,043 & $0,121-0,300$ \\
Temor & $4(4,71)$ & 0,022 & $0,012-0,116$ \\
Riesgo de Infección & $4(4,71)$ & 0,022 & $0,012-0,116$ \\
Mantenimiento ineficaz de la salud & $2(2,35)$ & 0,016 & $0,002-0,082$ \\
\hline
\end{tabular}

\section{Discusión}

Los Diagnósticos de enfermería más prevalentes correspondieron al Dominio de Cognición y percepción, específicamente Disposición para mejorar los conocimientos y Conocimientos deficientes; seguido del dominio de Actividad y Reposo, con el diagnóstico Disposición para mejorar el autocuidado; en el dominio de Promoción de la Salud se identificó el diagnóstico de Mantenimiento ineficaz de la salud; en el dominio de Afrontamiento y tolerancia al estrés con el diagnóstico de Temor y finalmente en el dominio de Seguridad y Protección el diagnóstico de Riesgo de infección.
En relación con los diagnósticos de enfermería disposición para mejorar los conocimientos y conocimientos deficientes presentan una prevalencia de $35,29 \%$ y $32,29 \%$ respectivamente, lo que significa que existe en la población asistente al programa una falta o escaso conocimiento, y el deseo de querer aprender sobre el proceso de la enfermedad y el procedimiento de la citología. Estudios orientados a determinar los conocimientos, prácticas y percepciones de las mujeres frente al cáncer de cérvix y la toma de la citología cervicouterina, coinciden en que una de las causas más comunes por las cuales las mujeres no se realizan la citología es el desconocimiento sobre el concepto de 
cáncer de cérvix, el procedimiento, la frecuencia y el objetivo principal de la prueba de la citología como tamizaje para la prevención y detección oportuna del cáncer de cérvix ${ }^{18-23}$. En contraste, un estudio realizado en Uganda en el 2017, en donde, el conocimiento general sobre los síntomas, los factores de riesgo y las medidas de prevención del cáncer de cérvix fue relativamente alto entre las mujeres entrevistadas, con un $88,2 \%$ (794), sin embargo, solo el $0,01 \%$ (7) conocía el procedimiento y la frecuencia recomendada para la citología cervicouterina ${ }^{24}$.

De acuerdo con lo anterior, el conocimiento se convierte en uno de los parámetros más importantes y determinantes para que la mujer demuestre una aptitud más favorable frente al interés de conocer y aprender, es decir, se trata de saber lo que ellas piensan y saben al respecto y como la información que ellas manejan es la que determina si realizan o no la toma de la citología cervicouterina ${ }^{18}$.

El diagnóstico Disposición para mejorar el autocuidado, se encuentra presente en este estudio en un $20,00 \%$ de las usuarias. El autocuidado es el comportamiento que integra una combinación de experiencias sociales y cognoscitivas y es aprendido a través de las relaciones interpersonales, de la comunicación y de la cultura; e incluye prácticas o destrezas aprendidas a través de toda la vida, de uso continuo y utilizadas por libre decisión con el propósito de fortalecer o reestablecer la salud y de prevenir la enfermedad ${ }^{25}$.

En lo que respecta a la baja capacidad de autocuidado el estudio realizado por Sosas Rosas, et al. establece que las prácticas de autocuidado de las mujeres ante el riesgo de cáncer cérvico uterino están íntimamente ligadas a su percepción de padecer la enfermedad, su historia personal, el contacto con otras mujeres que lo han padecido, su condición de género, su nivel educativo y el entorno de conocimientos que tengan por diversos medios ${ }^{26}$.

Para Gutiérrez, et al. la educación ha sido considerada un elemento influyente en adoptar medidas de autocuidado. La educación es la variable predictiva más íntimamente relacionada con el estado de salud de un individuo, población o comunidad, y constituye la senda para la implementación de conductas y actitudes favorables a la búsqueda de la salud encaminadas hacia el mantenimiento de la misma, lo cual conllevaría a un estilo de vida valioso y una mejor calidad de vida ${ }^{31}$. Es así, como una educación sanitaria insuficiente, acceso limitado a información, la falta de comunicación entre las mujeres y los proveedores de servicios de salud, y algunas barreras culturales, pueden estar relacionados con los niveles más bajos de conocimiento y prácticas de autocuidado hacia la detección del cáncer de cérvix ${ }^{20}$.

Un estudio cuyo objetivo fue analizar los conocimientos y las capacidades de autocuidado que tienen las mujeres para la prevención y control del cáncer cérvico uterino, evidenció que tener un nivel de autocuidado muy bajo en un $33 \%$, bajo autocuidado en $63 \%$ y sólo un $2,28 \%$ tiene las capacidades necesarias para cuidarse, resultados que relacionan el conocimiento sobre la utilidad de la citología con la realización de la misma, como una práctica de autocuidado en la mujer ${ }^{28}$. Resultados semejantes presentaron De la Cruz y Solís ${ }^{29}$, donde el 52,7\% de las mujeres del estudio, desconocen en términos globales la importancia de la citología, el $58,7 \%$ tienen una actitud favorable frente a la toma de la prueba, sin embargo, el $54 \%$ de las mujeres no asumen la prueba como una práctica de autocuidado de su salud. Los hallazgos citados, son comparables con nuestro estudio, en el que de 85 mujeres, el 55,29\% desconoce el objetivo principal de la citología y el $52,94 \%$ realizan la citología como una práctica anual de autocuidado en la salud femenina.

En los hallazgos encontrados en este estudio, en relación con los diagnósticos de Temor y Riesgo de infección con una prevalencia del 4,71\% cada uno y Mantenimiento ineficaz de la salud en un 2,35\%. El diagnóstico de temor según la NANDA, es una respuesta a la percepción de una amenaza que se reconoce conscientemente ${ }^{16}$; por lo tanto, el temor se percibe como una emoción de peligro, daño o dolor que podría influir en la adherencia de la mujer a los programas de tamizaje cervicouterino. Estudios han encontrado que el acceso a la citología está condicionado por factores inhibidores, entre ellos, el temor, el miedo, el dolor, el pudor, la vergüenza a la exploración y la angustia por conocer los resultados ${ }^{2,1,20,25,29-31}$.

El diagnóstico Mantenimiento ineficaz de la salud refleja la falta de interés, la incapacidad y el desconocimiento de la mujer para identificar, manejar o buscar ayuda para mantener el control de su salud en el ámbito sexual y reproductivo, y con ello, ser más vulnerable a presentar riesgo para adquirir infecciones vaginales y de trasmisión sexual, entre ellas el Virus del Papiloma Humano. 
Los resultados de esta investigación y de los estudios citados, invitan a que estudiantes y profesionales de enfermería sean capaces de pensar críticamente, diagnosticar en enfermería, identificar necesidades y escoger acciones adecuadas para solucionar los problemas en el cuidado de la salud sexual y reproductiva de la mujer. En este ámbito, la enseñanza del proceso de diagnosticar en enfermería, fortalece la participación activa del estudiante de forma que le permite profundizar y utilizar sus conocimientos para la transformación de la práctica de enfermería al disminuir la brecha entre la teoría y la práctica ${ }^{32}$.

La identificación de los diagnósticos de enfermería con mayor prevalencia en mujeres asistentes al programa de detección temprana de cáncer de cérvix, orienta al estudiante y profesional de enfermería, hoy y en el futuro, hacia la planificación y desarrollo de intervenciones educativas en salud más efectivas en las mujeres, que aborden principalmente la falta de conocimiento en cáncer de cérvix y un mayor nivel de conciencia y orientación de la importancia de la citología cervicouterina como una práctica del cuidado de sí misma y un compromiso frente a su salud sexual y reproductiva ${ }^{29,30}$; de manera que permita que las mujeres se apropien de una cultura del autocuidado en salud.

En este sentido, la participación de profesionales y estudiantes de enfermería es clave en la prevención y reducción de la incidencia y la carga de la enfermedad del cáncer de cérvix, en razón a que es el personal que tienen más contacto con las mujeres en las instituciones de salud y también porque las mujeres tienen en consideración la opinión y sus consejos en materia de salud ${ }^{20}$.

En los últimos años, se han desarrollado numerosos estudios orientados a identificar la prevalencia de diagnósticos de enfermería en diferentes contextos $^{12-15,33-35}$. Sin embargo, no se encontró evidencia científica, en relación a estudios orientados a identificar la frecuencia, incidencia o prevalencia de diagnósticos de enfermería en mujeres asistentes a programas de detección temprana de cáncer de cérvix. Por lo tanto, este estudio constituye un referente para futuras investigaciones, que pretendan identificar la calidad de la atención del programa de cáncer de cérvix en instituciones de salud, visto desde el componente de educación en salud, la comunicación terapéutica entre personal y las usuarias y la adherencia al tamizaje cérvico uterino.
Además, este tipo de estudios permitirá fortalecer la aplicación del lenguaje estandarizado en la práctica de enfermería, servirá como estrategia para la enseñanza en la toma de decisiones en escenarios comunitarios, e implementar planes de cuidados orientados a fortalecer en la mujer las necesidades de educación en salud y el compromiso hacia su autocuidado, aplicando intervenciones y resultados propios de enfermería, lo cual contribuye al desarrollo del conocimiento disciplinar.

\section{Conclusiones}

En esta investigación se han identificado los diagnósticos de enfermería más frecuentes en las usuarias asistentes al programa de detección temprana de cáncer de cérvix, convirtiéndose en un reto no solo para los profesionales de enfermería sino para los estudiantes del programa de enfermería de la Universidad Industrial de Santander y de cualquier otra institución de educación superior con programas de enfermería; para llevar a cabo planes de cuidado estandarizados a partir del proceso de atención de enfermería, que les permitan trabajar en el desarrollo de una atención integral y efectiva desde el contexto académico hasta el profesional, es decir, aportar en la disminución de la prevalencia de los diagnósticos encontrados que sin duda repercuten en el conocimiento y la capacidad de autocuidado de las mujeres hacia promoción de la salud y la prevención y detección oportuna del cáncer de cérvix.

Los resultados de esta investigación se convierten en una herramienta indispensable en el cuidado de la salud de la mujer tanto en la práctica como en la docencia. Además, constituye un referente para futuras investigaciones orientadas a la validación científica de estos diagnósticos, y estudios de intervención de enfermería de manera que permitan demostrar la eficacia y la efectividad de las intervenciones de enfermería en los diagnósticos más prevalentes reportados en este estudio.

\section{Agradecimientos}

Las autoras agradecen al grupo de estudiantes de tercer nivel de enfermería de la Universidad Industrial de Santander, quienes recolectaron los datos para el presente estudio mediante la aplicación del formato del Sistema de Información de Prácticas Clínicas y Comunitarias Estudiantiles- SIPCE, durante el desarrollo de sus prácticas formativas. 


\section{Consideraciones éticas}

En la realización de esta investigación se tuvo en cuenta las consideraciones éticas planteadas en la Resolución No. 08430 de 1993 del Ministerio de Salud y Protección Social de Colombia, por la cual se establecen las normas científicas, técnicas y administrativas para la investigación en salud; y clasifica a esta investigación sin riesgo para las participantes, ya que no se realizó ninguna intervención o modificación intencionada de las variables biológicas, fisiológicas, sicológicas o sociales de las participantes.

Adicionalmente se garantizó la confidencialidad de la información y el respeto de los principios éticos contemplados en la Ley 911 de 2004.

\section{Conflicto de interés}

Los autores manifiestan no tener ningún conflicto de interés en el presente estudio

\section{Referencias}

1. Rizalar S, Aydin Avci I, Żołądkiewicz P, Altay B, Moraczewska I. University Students' Awareness of Breast and Cervical Cancers: A Comparison of Two Countries and Two Different Cultures. J Breast Heal. 2017; 13(2): 77-82. doi: 10.5152/tjbh.2017.3117

2. Aranguren Pulido L, Burbano Castro J, González J, Mojica Cachope A, Plazas Veloza D, Prieto Bocanegra M. Barreras para la prevención y detección temprana de cáncer de cuello uterino. Inv Enferm Imagen Desarr. 2017; 19(2): 129-144. doi: https://doi.org/10.11144/Javeriana.ie19-2.bpdt

3. Hernández DM, Apresa T, Patlán RM. Panorama epidemiológico del cáncer cervicouterino. Rev Med Inst Mex Seguro Soc. 2015; 53 Supl 2: S154-161.

4. Muñoz N, Bravo LE. Epidemiology of cervical cancer in Colombia. Salud Pública Mex. 2014; 56(5): 431-439. doi: https://doi.org/10.25100/ cm.v43i4.1269

5. Ministerio de Salud y Protección Social. Instituto Nacional de Salud Informe de cáncer de mama y cuello uterino en Colombia, 2018. Versión Mayo 31 de 2019.

6. Ministerio de Salud - Dirección General de Promoción y Prevención. Norma Técnica para la Detección Temprana del Cáncer de Cuello Uterino y Guía de Atención de Lesiones Preneoplásicas de Cuello Uterino. 2000.
7. Ministerio de Salud y Protección Social. Guía de Práctica Clínica para la detección y manejo de lesiones precancerosas de cuello uterino. Guía para pacientes y cuidadores. Colombia 2014.

8. Ministerio de Salud y Protección Social, Profamilia. Encuesta Nacional de Demografía y Salud. Tomo II. Componente de Salud Sexual y Reproductiva. Capítulo 12. Detección temprana del cáncer. 2015; p.235.

9. Ministerio de Salud y Protección Social, Instituto Nacional de Cancerología, E.S.E. Plan Decenal para el Control del Cáncer en Colombia, 2012-2021. Bogotá D.C. 2012; p 58-59.

10. Ministerio de Salud y Protección Social, Fondo de Población de las Naciones Unidas. Protocolos para la atención de enfermería a la salud sexual y reproductiva de la mujer. Bogotá DC. 2014; p.22.

11. Lagoueyte MI. El cuidado de enfermería a los grupos humanos. Rev Univ Ind Santander Salud. 2015; 47(2): 209-213.

12. Gómez J, Mayorga ME, Pérez J, Rojas Lyda, Orozco LC, Camargo F. Prevalencia de diagnósticos de enfermería en personas con VIH/SIDA. Enferm Glob. 2013; 12(32): 1-10.

13. Gallegos M, Parra M. Diagnósticos de enfermería identificados en pacientes con cáncer. Rev CONAMED. 2008; 13, Suppl 1: 12-16.

14. Pinilla E; Pérez Y. Prevalecia de diagnósticos de enfermería en escolares desplazados víctimas de la violencia social e intrafamiliar. Rev Univ Ind Santander Salud. 2009; 41(2): 149-156.

15. Rodríguez M, Rincón Y, Reyes A, Fajardo MT, Orozco LC, Camargo FA. Prevalencia de los diagnósticos de enfermería en niños que reciben tratamiento oncológico. Enferm Global. 2012; 11(3): 77-85. doi: http://dx.doi.org/10.4321/S169561412012000300005

16. Herdman TH, Kamitsuru S. NANDA Internacional. Diagnósticos Enfermeros. Definiciones y Clasificación 2015-2017. 10 ed. Barcelona: Elsevier; 2015.

17. StataCorp. 2015. Stata 14 Base Reference Manual. College Station, TX: Stata Press.

18. Sanabria N. Conocimientos, prácticas y percepciones de mujeres sobre la citología vaginal. Enfermería en Costa Rica. 2012; 33(2): 82-89.

19. López C, Calderón M, González M. Conocimientos, actitudes y prácticas respecto al cáncer de cuello uterino de mujeres entre 14 y 49 años de un barrio de la comuna 10 de la ciudad de Armenia, Colombia. Rev. Méd. Risaralda. 2013; 19 (1): 14-20. 
20. Liu T, Li S, Ratcliffe J, Chen G. Assessing Knowledge and attitudes towards cervical cancer screening among rural women in Eastern China. Int. J. Environ Res Public Health. 2017; 14(9): 967. doi: $10.3390 /$ ijerph 14090967

21. Okunowo A, Daramola E, Soibi-Harry A, Ezenwankwo FC, Kuku J, Okunade K, et al. Women's knowledge of cervical cancer and uptake of Pap smear testing and the factors influencing it in a Nigerian tertiary hospital. J Cancer Res Pract. 2018. doi: https://doi.org/10.1016/j.jcrpr.2018.02.001

22. Di J, Rutherford S, Wu J, Song B, Ma L, Chen J, et al. Knowledge of Cervical Cancer Screening among Women across Different Socio-Economic Regions of China. PLoS ONE. 2015. doi: https:// doi.org/10.1371/journal.pone.0144819

23. Thapa N, Maharjan M, Petrini M, Shah R, Shah S, Maharjan N, et al. Knowledge, attitude, practice and barriers of cervical cancer screening among women living in mid-western rural, Nepal. J Gynecol Oncol. 2018; 29 (4): e57. doi: 10.3802/jgo.2018.29.e57

24. Mukama T, Ndejjo R, Musabyimana A, Halage A, Musoke D. Women's knowledge and attitudes towards cervical cancer prevention: a cross sectional study in Eastern Uganda. BMC Women's Health. 2017;17(9). doi: 10.1186/s12905-017-0365-3

25. Tamayo L, Chávez M, Henao L. Cáncer de cuello uterino: más allá de lo que es la percepción de las mujeres de Antioquia (Colombia) y Colima (México), 2008. Rev Fac Nac Salud Pública 2009; 27(2): 177-186.

26. Sosa M, López N, Torres M. Sentido y significancia de prácticas de autocuidado ante el riesgo de cáncer cérvico-uterino en mujeres mexicanas sanas. Revista Enfermería ENEO-UNAM. 2008; 5 (4): 10-16. doi: https://doi.org/10.22201/eneo.23958421e.2008.4.377

27. Gutiérrez C, Peña C, Zamorano D. Medidas de autocuidado y genotipificación del virus papiloma humano en mujeres de la unidad de patología cervical, Hospital Carlos Van Buren. Rev Chil Salud Pública 2016; 20(1): 19-28. doi: 10.5354/07195281.2016.39292

28. Díaz J, Soria C, Gutiérrez C, Esparza R, Nerí I, Uribe E. Capacidades de Autocuidado del Cáncer Cérvico Uterino en Mujeres Obreras. Sistema educativo estatal. Gobierno del Estado. GobBC. 46-56.

29. De la Cruz T, Solís A. Conocimientos, actitudes y prácticas sobre papanicolaou en mujeres del pueblo joven $1^{\circ}$ De mayo, Nuevo Chimbote, 2014. In Crescendo. Ciencias de la Salud. 2017; 4(1): 159-172.
30. Urrutia M, Araya A, Poupin L. ¿Por qué las mujeres no se toman el papanicolaou? Respuestas entregadas por los profesionales del programa cáncer cervicouterino- auge del servicio de salud metropolitano sur oriente. Rev. Chil. Obstet. Ginecol. 2010; 75(5): 284-289. doi: http://dx.doi. org/10.4067/S0717-75262010000500002

31. Garcés I, Rubio D, Scarinci I. Factores asociados con el tamizaje de cáncer de cuello uterino en mujeres de nivel socioeconómico medio y bajo en Bogotá, Colombia. Rev Fac Nac. Salud Pública. 2012; 30(1): 7-16.

32. Brandão de Carvalho A, Venícios de Oliveira M. Diagnóstico de enfermería: estrategia educativa fundamentada en el aprendizaje basado en problemas. Rev Latino-Am. Enfermagem. 2011; 19(4).

33. Padilla CI, Romero SL, Camargo FA, Bonilla AP. Diagnósticos de enfermería al alta hospitalaria en personas con Síndrome Coronario Agudo. Med UNAB. 2017; 20 (1):19-27.

34. Pinilla E, Orozco LC, Camargo FA, Alfonso EP, Peña ER, Villabona LN, et al. Lactancia materna ineficaz: prevalencia y factores asociados. Rev Univ Ind Santander Salud. 2011; 43(3): 271-279.

35. Rivas JG, Álvarez ME, Cruz C, Martínez FJ, Aguilera M, Ibánez DR. Etiquetas diagnósticas en pacientes hospitalizadas con diagnóstico de cáncer cérvico-uterino. Rev CONAMED. 2008; 13 supl 2: 23-29. 